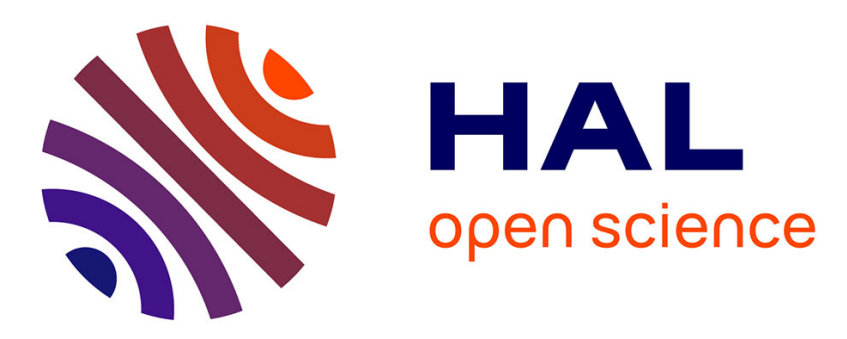

\title{
Quasi-condensation of bilayer excitons in a periodic potential
}

Camille Lagoin, Stephan Suffit, Kenneth West, Kirk Baldwin, Loren Pfeiffer, Markus Holzmann, Francois Dubin

\section{To cite this version:}

Camille Lagoin, Stephan Suffit, Kenneth West, Kirk Baldwin, Loren Pfeiffer, et al.. Quasicondensation of bilayer excitons in a periodic potential. Physical Review Letters, 2021, 126 (6), pp.067404. 10.1103/PhysRevLett.126.067404 . hal-03368562

\section{HAL Id: hal-03368562 \\ https://cnrs.hal.science/hal-03368562}

Submitted on 6 Oct 2021

HAL is a multi-disciplinary open access archive for the deposit and dissemination of scientific research documents, whether they are published or not. The documents may come from teaching and research institutions in France or abroad, or from public or private research centers.
L'archive ouverte pluridisciplinaire HAL, est destinée au dépôt et à la diffusion de documents scientifiques de niveau recherche, publiés ou non, émanant des établissements d'enseignement et de recherche français ou étrangers, des laboratoires publics ou privés. 


\title{
Quasicondensation of Bilayer Excitons in a Periodic Potential
}

\author{
Camille Lagoin, ${ }^{1}$ Stephan Suffit, ${ }^{2}$ Kenneth West, ${ }^{3}$ Kirk Baldwin, ${ }^{3}$ Loren Pfeiffer, ${ }^{3}$ Markus Holzmann, ${ }^{4}$ and François Dubin ${ }^{1}$ \\ ${ }^{1}$ Institut des Nanosciences de Paris, CNRS and Sorbonne Université, 4 pl. Jussieu, 75005 Paris, France \\ ${ }^{2}$ Laboratoire de Materiaux et Phenomenes Quantiques, Universite Paris Diderot, 75013 Paris, France \\ ${ }^{3}$ PRISM, Princeton Institute for the Science and Technology of Materials, Princeton University, Princeton, New Jersey 08540, USA \\ ${ }^{4}$ Univ. Grenoble Alpes, CNRS, LPMMC, 38000 Grenoble, France
}

(Received 18 September 2020; accepted 14 January 2021; published 12 February 2021)

\begin{abstract}
We study two-dimensional excitons confined in a lattice potential, for high fillings of the lattice sites. We show that a quasicondensate is possibly formed for small values of the lattice depth, but for larger ones the critical phase-space density for quasicondensation rapidly exceeds our experimental reach, due to an increase of the exciton effective mass. On the other hand, in the regime of a deep lattice potential where excitons are strongly localized at the lattice sites, we show that an array of phase-independent quasicondensates, different from a Mott insulator, is realized.
\end{abstract}

DOI: 10.1103/PhysRevLett.126.067404

The phase diagram of bosonic particles exploring a lattice potential is commonly described in terms of two essential parameters: the interparticle interaction strength $U$ giving the increase of energy when two particles occupy the same lattice site, and the particle tunneling strength $J$, determining the hopping of a particle to a neighboring site [1-3]. Within the minimal Bose-Hubbard model, two antagonist regimes emerge at zero temperature. When tunneling is dominant, a superfluid showing phase ordering over spatially distinct sites can be reached, whereas above a critical ratio $U / J$ tunneling is suppressed and bosons remain fixed to their lattice site. This results in an incompressible phase, a socalled Mott insulator, where phase coherence extending over various lattice sites is absent. Typically, varying the lattice depth or the lattice period, $U$ and $J$ are modified and the phase diagram is accurately explored $[2,3]$.

Seminal experiments conducted with ultracold atomic gases confined in optical lattices have provided model studies of the Bose-Hubard model, initially in three dimensions, and more recently, for effectively two- and one-dimensional systems [3]. In the solid state, large efforts have also been dedicated to the physics of the twodimensional Bose-Hubbard Hamiltonian, in particular for quantum simulation perspectives. To this aim, promising candidates include long-lived dipolar excitons of semiconductor bilayers. Such excitons are marked by a spatial separation imposed between Coulomb-bound electrons and holes. They are possibly engineered in coupled GaAs quantum wells [4] or in van der Waals assemblies of transition metal dichalcogenides monolayers [5]. In the former case, excitons may be subject to an artificial lattice potential engineered by electrostatic gates $[6,7]$ while in the latter case they naturally explore a periodic moiré potential [8-10]. For both systems, the Bose-Hubbard model is expected to provide an accurate low-energy description.
To explore the collective phases accessible to dipolar excitons, GaAs double quantum wells provide an experimental toy model system, since excitons are then possibly manipulated in a regime of homogeneous broadening [11], and spatially confined in on-demand potential landscapes [12-17]. Using these degrees of freedom, we have recently mapped out the quasicondensation crossover of bilayer excitons in boxlike trapping potentials $[11,18,19]$, determining the excitons' equation of state and density fluctuations, and correlated these to the degree of spatial and temporal coherence at subkelvin temperatures.

Here, we report experiments characterizing the quasicondensation of GaAs bilayer excitons in a microscopic lattice potential. We show that a quasicondensate is destroyed above a threshold lattice depth around a fraction of the chemical potential. We attribute the suppression of coherence to the renormalization of the exciton effective mass by the lattice potential which rapidly increases the critical phase-space density of quasicondensation. Above a threshold lattice depth, cold excitons enter then a normal phase, but without yet being spatially localized at the lattice sites. The localized regime is in fact obtained for lattice depths large compared to the chemical potential. In this regime, we show that an array of phase incoherent quasicondensates localized at each site is possibly formed at large filling factors. We underline that this realization differs from a Mott insulator.

We study a square artificial lattice with spatial period $L=3 \mu \mathrm{m}$, at a bath temperature $T_{b}$ set to $340 \mathrm{mK}$ (see Ref. [6] for the characterization of our device). Indirect excitons are injected in the lattice potential using a 100-ns-long laser excitation, repeated at $1.5 \mathrm{MHz}$, resonant with the direct exciton absorption. In the following, we focus on excitonic properties at a fixed average density $n \sim 2 \times 10^{10} \mathrm{~cm}^{-2}$, obtained $150 \mathrm{~ns}$ after extinction of the 

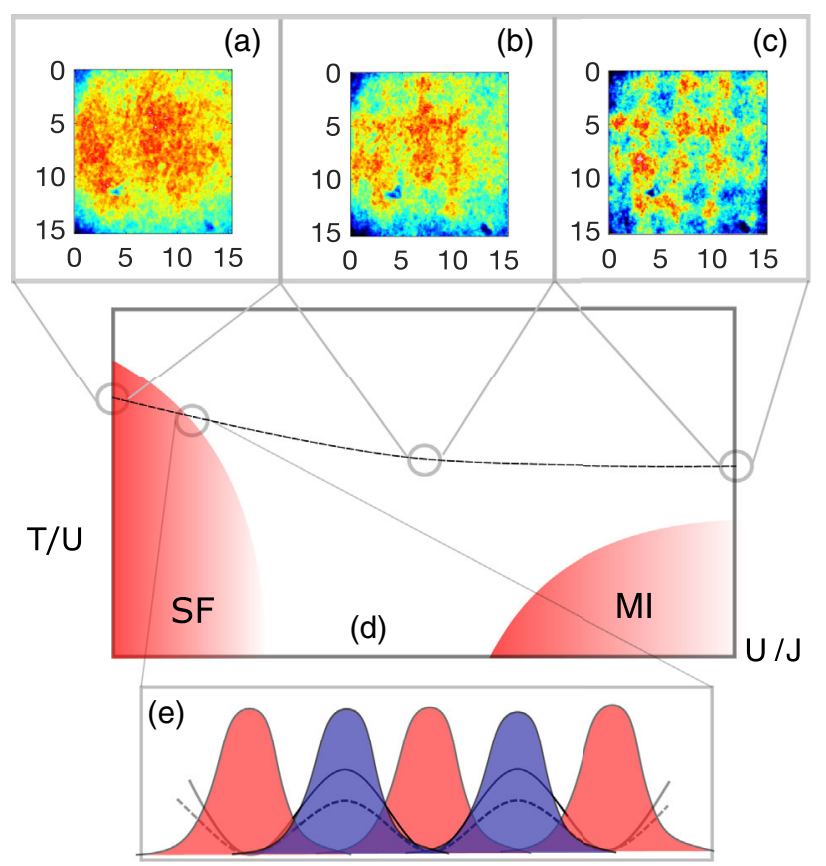

FIG. 1. (a)-(c) Real images of the photoluminescence for $V_{0}=0$ (a), $1.5 \mathrm{meV}$ (b), and $3 \mathrm{meV}$ (c). Scales are given in $\mu \mathrm{m}$. (d) Schematic phase diagram for the two-dimensional BoseHubbard model, as a function of the on-site interaction $U$, the tunneling strength $J$, and the temperature $T$. At nonzero temperature superfluid (SF) and Mott-insulating (MI) phases are separated by a normal phase where physical properties are classical. Our experiments, carried out at a fixed average density, explore the parameter space along the dashed curved line. (e) At the crossover between the SF and normal phases noncondensed excitons (blue) are mostly located around the lattice barrier. This effectively increases the lattice depth (solid line) compared to the native one (dashed line). Quasicondensed excitons are depicted in red.

laser excitation for an average power set to $1.5 \mu \mathrm{W}$. The phase-space density $D$ is then around 12 corresponding to a chemical potential $\mu$ of about $0.8 \mathrm{meV}$, i.e., well above the critical value $D_{c} \sim 8$ for exciton quasicondensation [11]. Thus, we explore how the lattice depth influences quantum statistical signatures, by continuously varying the height of the potential barrier $V_{0}$ separating the lattice sites [6].

Figure 1(d) illustrates the phases potentially accessible in our experiments: for small $U / J$, realized in the limit of vanishing lattice depth, a quasicondensate phase marked by quasi-long-range order in the spatial and temporal coherence is expected $[2,20]$. On the other hand, for a deep lattice potential, i.e., for large $U / J$, one possibly enters the regime where a Mott-insulating phase (MI), defined by the same fixed number of excitons per lattice site, fluctuations being suppressed by interaction [2], is energetically favorable. We then note that MI phases are protected since their minimum excitation, resulting from an exciton hoping between two lattice sites, has an energy cost equal to $U=\left(\hbar^{2} / 4 \pi m_{X} a_{0}^{2}\right) \tilde{g}, m_{X}$ being the exciton's effective mass, $a_{0}$ describing the spatial extension of the exciton's wave function in lattice sites, while $\tilde{g} \sim 4$ is a dimensionless parameter quantifying dipolar interactions between excitons [11]. For our experimental conditions we deduce that $U \sim k_{B} T_{b}$. Then MI phases are not accessible due to thermal excitations. Moreover, let us note that for our device we only have access to high-filling factors, with around 100 excitons per site.

We now turn to discuss our experimental results in these different regimes. Let us start with the quasicondensate regime at vanishing $U / J$, by setting $V_{0}$ to 0 . In this situation, we studied the degree of spatial and temporal coherence of the photoluminescence radiated by our device using a MachZehnder interferometer where the photoluminescence field $\psi$ is recombined with itself, after a variable time delay $\tau$, or a spatial displacement $\mathbf{r}$ is introduced. Measuring the interference contrast at the interferometer output, we directly deduced the amplitude of the first-order correlation function $\left|g^{(1)}(\mathbf{r}, \tau)\right| \sim\left|\left\langle\psi^{*}\left(\mathbf{r}_{i}, t\right) \psi\left(\mathbf{r}_{i}+\mathbf{r}, t+\tau\right)\right\rangle_{r_{i}, t}\right|$. Here $\langle\cdots\rangle_{r_{i}, t}$ denotes the average over $t$, i.e., over the number of realizations accumulated to produce one interferogram, as well as the average over the positions $\mathbf{r}_{i}$ where the fringe contrast is evaluated, typically of the order of $10 \mu \mathrm{m}$ to compute the contrast from 3 fringes [Figs. 2(a) and 2(b)].

Figure 2(c) shows the variation of the photoluminescence first-order time correlation function $\left|g^{(1)}(0, \tau)\right|$. We note that initially it decays exponentially with a time constant $\tau_{c} \sim 4 \mathrm{ps}$, before a slower decay is found for $4 \lesssim \tau \lesssim 7$ ps. Very recently we have reported such behavior and shown that the initial exponential decay reveals the contribution of noncondensed excitons through the inelastic two-body collisional rate. Indeed, the exciton-photon coupling is linear so that the coherence of optically bright excitons is imprinted in the photoluminescence [21]. Thus, we find here an exciton's collisional rate, $1 / \tau_{c}$, in good agreement with our previous studies [11]. On the other hand, the slowly decaying part for $\tau \gtrsim \tau_{c}$ marks the contribution of quasicondensed excitons, which exhibit an algebraically decaying time coherence $\tau^{-\eta}$ [20]. Importantly, here we find that $\eta \sim 0.25$, which is compatible with the value predicted at criticality by the Berezinskii-Kosterlitz-Thouless theory [22,23]. Let us then note that our studies are realized for an exciton phase-space density $D \sim 12$, very close to Ref. [11] where a very similar exponent was deduced.

We further studied the degree of spatial coherence by setting our interferometer such that $\|\mathbf{r}\| \sim 2 \mu \mathrm{m}$ and $\tau=0$. This spatial separation is over an order of magnitude beyond the classical limit set by the thermal de Broglie wavelength, and 2 times larger than our optical resolution. Figure 2(d) reveals that we observe interference fringes signaling the buildup of quasi-long-range spatial coherence. In these experiments the interference contrast amounts to $23 \%$, compared to $75 \%$ at the spatial autocorrelation. From this difference we deduce that $1 / 3$ of the 

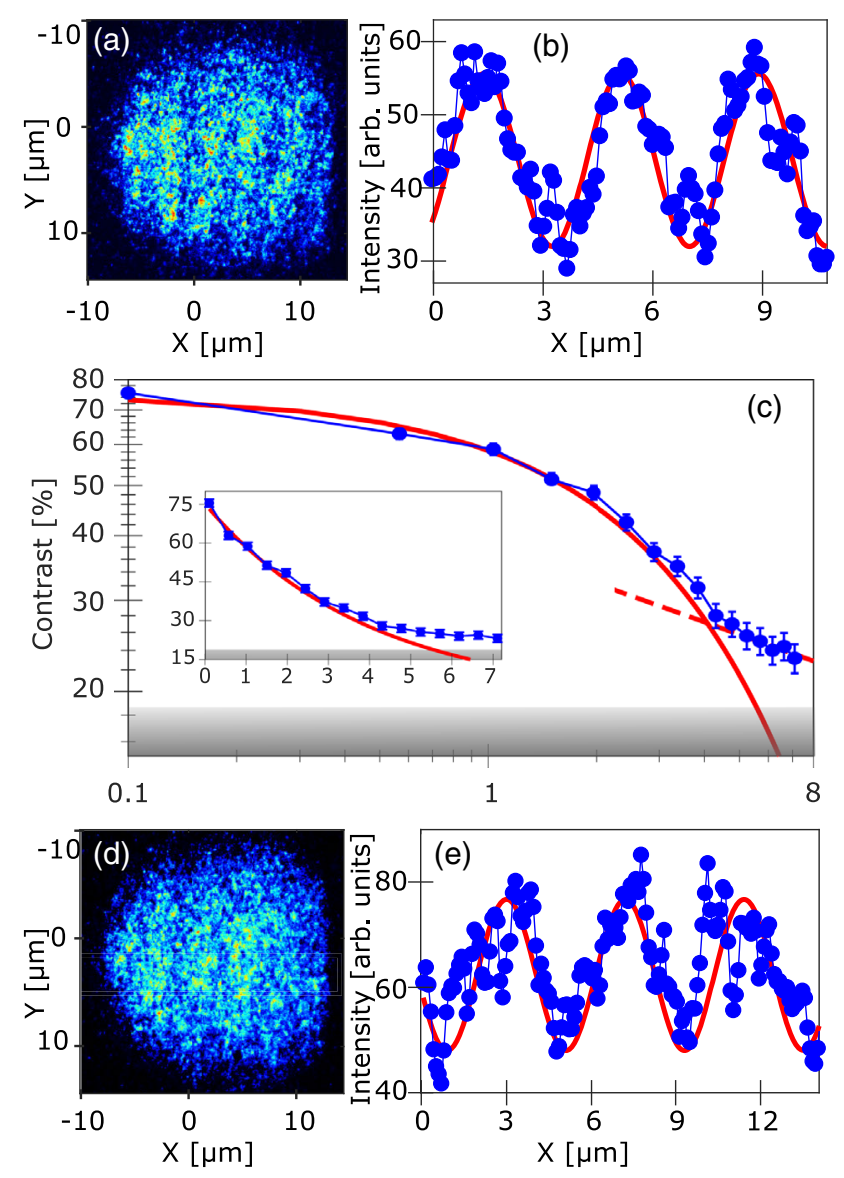

FIG. 2. (a) Interference pattern measured for $V_{0}=0$ and for $(\mathbf{r}=0, \tau=4.2 \mathrm{ps})$, together with the profile evaluated at the center of the image (b) leading to $\left|g^{(1)}\right|=28 \%$. (c) Decay of the first-order time coherence $\left|g^{(1)}(0, \tau)\right|$ as a function of the time delay $\tau$. Experimental data are depicted by the solid blue points, while the red line shows an exponential decay with a characteristic time constant of $4 \mathrm{ps}$. The dashed line shows an algebraic time decay $\tau^{-\eta}$ with $\eta=0.25$. The inset presents the same measurements in linear scale and the gray shaded area marks the limit of our experimental precision. (d) Spatial interference of the photoluminescence measured for $(\|\mathbf{r}\|=2 \mu \mathrm{m}, \tau=0)$, together with the interference profile evaluated at the center of the image (e). Thus we deduce an interference contrast $\left|g^{(1)}\right|=23 \%$. The interference period is set to $3 \mu \mathrm{m}$ in (a) and (b) and to $4.5 \mu \mathrm{m}$ in (d) and (e).

optically bright excitons' population contributes to the quasicondensate [24]. In previous works, we had shown that a quasicondensate also includes optically dark excitons, that constitute around $2 / 3$ of the total exciton population at $340 \mathrm{mK}$ [25]. However, the quasicondensed fraction of dark excitons, $58 \% \pm 42 \%$, is extracted with a low accuracy since these are optically inactive so that their quantum statistical distribution is not measurable precisely. Thus, the overall fraction of quasicondensed excitons amounts roughly to $50 \%$, with a possible shift by $\pm 25 \%$ due to our uncertainty when extracting the fraction of
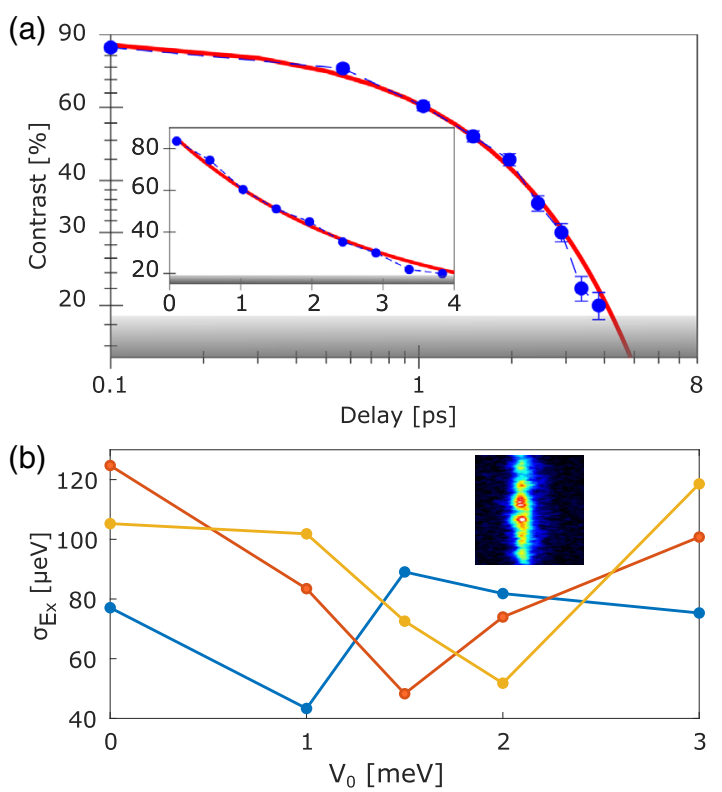

FIG. 3. (a) Decay of the first-order time coherence for $V_{0}=$ $3 \mathrm{meV}$ in log-log scale. Experimental data are displayed by the blue points while the red line shows an exponential decay with a characteristic time equal to $2.8 \mathrm{ps}$. The inset provides the same results in linear scale while the gray area underlines the limit set by the signal-to-noise ratio. (b) Standard deviation of the photoluminescence energy measured at the position of 3 adjacent lattice sites as a function of the lattice depth $V_{0}$. Each measurement is evaluated from an ensemble of 10 realizations all performed under the same experimental conditions. The inset provides a spatially resolved spectrum where the emission from individual lattice sites is identified [6].

quasicondensed dark excitons. These magnitudes well reproduce the ones we deduced in electrostatic traps for similar densities [18], in good agreement with path integral Monte Carlo calculations [26]. Also, note that the amplitude of $\left|g^{(1)}\right|$ is of the same order for $(\|\mathbf{r}\| \sim 2 \mu \mathrm{m}, \tau=0)$ and ( $\mathbf{r}=0, \tau \sim 6 \mathrm{ps}$ ). This matching is expected since in both cases only the fraction of quasicondensed bright excitons contributes to the interference signal.

We now turn to the opposite regime where a deep lattice potential is imprinted, $V_{0}=3 \mathrm{meV} \gg \mu$. In this situation Fig. 1(c) shows that excitons are strongly localized in the lattice sites which have a characteristic spatial extension of about $1 \mu \mathrm{m}$. This results in a modulation of the photoluminescence intensity, of around $20 \%$ along the horizontal axis of our device, with a $3 \mu \mathrm{m}$ period [6]. To study spatially extended time coherence in the photoluminescence, we studied $\left|g^{(1)}(0, \tau)\right|$ fixing the interference period to $4.5 \mu \mathrm{m}$, as in the measurements shown in Figs. 2(d) and 2(e). Thus, the interference period is clearly distinguished from the one of the spatial modulation of the photoluminescence intensity due to the lattice potential. Figure 3(a) shows the variation of the interference contrast with $\tau$, which, unlike in Fig. 2(c), is reduced to an exponential decay with a characteristic time of around 
3 ps. We then note that for $V_{0}=3 \mathrm{meV}$ a slightly higher rate of inelastic collisions is deduced compared to $V_{0}=0$. We attribute this difference as the manifestation of an increased concentration of excess carriers [27] when we impose a large difference between the potentials applied onto our gate electrodes, as necessary to engineer a deep lattice potential. As a result, excitons suffer additional collisions with free carriers; nevertheless, the photoluminescence is homogeneously broadened in this regime as well.

Figure 3(a) shows that in a deep lattice potential cold excitons do not exhibit any algebraically decaying time coherence, and therefore lack quasi-long-range order $[11,20]$. This implies that the phase between excitons confined in each lattice site is randomly distributed. We actually expected such a conclusion since the coupling between the lattice sites, controlled by the tunneling coefficient $J$, decreases exponentially with the barrier height, like $e^{-\left(V_{0} / E_{r}\right)^{1 / 2}}$, where $E_{r}=\pi^{2} \hbar^{2} /\left(2 m L^{2}\right) \sim 0.2 \mu \mathrm{eV} \mathrm{[2].} J$ is then vanishingly small for $V_{0} \sim 3 \mathrm{meV}$. Moreover, the schematic phase diagram shown in Fig. 1 recalls that for $V_{0}=3 \mathrm{meV}$ a Mott-insulating phase is potentially accessible. As mentioned earlier, this is unlikely in our experiments carried out at a temperature comparable to the energy gap protecting Mott phases. To verify this expectation we studied the variation of density fluctuations in the lattice sites as a function of the lattice depth. Indeed a Mott insulator is signaled by the same fixed number of particles in each site, so that density fluctuations are theoretically vanishingly small. For dipolar excitons density fluctuations are directly accessed by the energy of the photoluminescence which is governed by the strength of repulsive dipolar interactions between excitons [28-30]. Figure 3(b) compares the standard deviation of the photoluminescence energy $\sigma_{E_{X}}$ for 3 neighboring lattice sites as a function of $V_{0}$. Overall, it shows that the photoluminescence energy is highly stable in our experiments, since it varies by only around $80 \mu \mathrm{eV}$. However, we do not observe any clear dependence of $\sigma_{E_{X}}$ over $V_{0}$, and thus no sign of a Mott-insulating phase is detected in the deep lattice regime. Moreover, we note that Fig. 3(b) indicates strongly that thermodynamic equilibrium is reached across the lattice potential since density fluctuations do not depend on $V_{0}$ [31].

Figures 2 and 3 show that extended temporal coherence is destroyed when we pass from the regime where excitons are delocalized in the lattice potential $\left(V_{0}=0\right)$ to the one where they are strongly localized $\left(V_{0}=3 \mathrm{meV}\right)$. To quantify this transition, we have measured the degree of temporal coherence as a function of the lattice depth, setting $\tau$ to 4.7 ps. Starting from a flat potential $\left(V_{0}=0\right)$, Fig. 4(a) shows that $\left|g^{(1)}\right|$ decays very rapidly when $V_{0}$ is increased by a few $100 \mu \mathrm{eV}$. Actually, $\left|g^{(1)}\right|$ is reduced to the amplitude it would reach by decaying exponentially at a rate $\tau_{c}$ when $V_{0} \sim 0.2 \mathrm{meV}$. This magnitude signals that time coherence is governed by inelastic two-body collisions only,

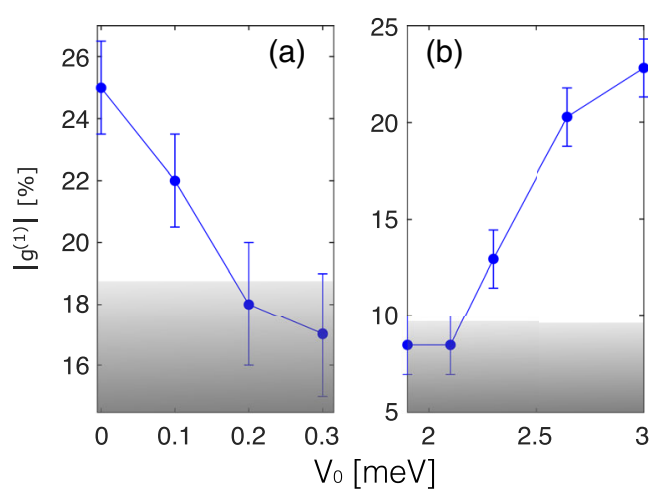

FIG. 4. (a) First-order time coherence as a function of the barrier height $V_{0}$ in the weak lattice limit. The contrast measures the amplitude of the first-order time correlation function $\left|g^{(1)}(0,4.7 \mathrm{ps})\right|$. (b) Local time coherence in the deep lattice limit. Here the contrast measures the amplitude of the intensity modulation along a bright interference fringe, i.e., along the weaker confining direction. The shaded areas display our instrumental resolution limited by the signal-to-noise ratio in these measurements. Experiments have all been performed at a bath temperature $T_{b}=340 \mathrm{mK}$.

manifesting that the fraction of quasicondensed excitons has vanished.

The loss of coherence in a periodic potential is usually discussed in terms of arrays of condensates in single lattice sites connected by a Josephson coupling describing quantum tunneling [31-35]. The latter decreases exponentially with the difference between the barrier height and the chemical potential [36]. In our strongly interacting system, the barrier height seen by the quasicondensate must also include the interaction with noncondensed excitons. Indeed, unlike in a flat confining potential, in a lattice noncondensed excitons are mostly localized around the barriers separating accessible sites [37] [see Fig. 1(e)]. This periodic arrangement minimizes the system energy and leads to an effective barrier height $V_{0}^{*} \approx V_{0}+2(\hbar / m) \tilde{g}\left(n_{n c}^{(\max )}-n_{n c}^{(\min )}\right)$. Here, $n_{n c}^{(\max / \min )}$ are the maximum/minimum local density of noncondensed excitons. As previously discussed, their difference is of the order of half of the total population. Accordingly, we deduce that $V_{0}^{*} \sim \mu$ for $V_{0} \sim 0.2 \mathrm{meV} \ll \mu$, and a transition from a coherent condensate to incoherent array of microcondensates can be expected for our parameter space [32,33]. Alternatively, at finite temperatures the loss of coherence can be understood in terms of a renormalized effective mass $m_{X}^{*}=m_{X} /\left(1-V_{0}^{* 2} / 2 \mu^{2}\right)$ for small lattice amplitudes $\left(V_{0} \ll \mu\right)$ [38]. For $V_{0}^{*} \sim \mu$ one has $m_{X}^{*} \sim 2 m_{X}$ so that the effective critical phasespace density for quasicondensation increases by a factor of 2 , from 8 to 16 . It then exceeds our experimental value $D \sim 12$, driving a transition from a coherent (quasiuniform) condensate to an incoherent normal gas, as observed in Fig. 4(a). 
The experiments reported in Fig. 4(b) allow us to distinguish the two previous scenarios. There, we extracted the exciton time coherence locally, i.e., in the lattice sites, by ensuring that one bright interference fringe coincided with one row of lattice sites. For each value of $V_{0}$ we then measured the intensity modulation along this particular bright fringe $C_{i}$, and compared it to the bare modulation of the photoluminescence in real space $C_{m}$ due to the localization in the lattice. Thus, we directly deduce the average amplitude of $\left|g^{(1)}\right|$ for the photoluminescence radiated by the lattice sites only. It reads $\left|g^{(1)}\right|=2\left(C_{i}-C_{m}\right) /\left[\left(1-C_{i}\right)\left(1+C_{m}\right)\right]$, since the emission between the lattice sites does not yield any measurable interference signal for $\tau=4.7$ ps [Fig. 3(a)]. Figure 4(b) reveals then that the interference contrast is vanishing for $V_{0} \lesssim 2 \mathrm{meV}$, the contrast increasing thereafter rapidly with $V_{0}$. For $V_{0}=3 \mathrm{meV}$ it reaches an amplitude similar to the one for $V_{0} \sim 0$. Accordingly, we deduce that for $V_{0} \lesssim 2 \mathrm{meV}$ excitons lack extended temporal coherence, whereas coherence builds up for $V_{0} \gtrsim 2 \mathrm{meV}$ providing a signature of microscopic quasicondensates in the lattice sites for $V_{0}=3 \mathrm{meV}[11,20]$. As shown in Fig. 3, these coherent exciton droplets are independent from one another, i.e., with no defined phase relation. Figure 4 shows that the transition between this array of droplets and the extended quasicondensate $\left(V_{0} \sim 0\right)$ passes then through a normal incoherent exciton gas which is not localized by the lattice potential (see Ref. [6]). Such transition does not correspond to the framework of Josephson coupled array of condensates, but rather suggests that coherence is destroyed due to a renormalization of the exciton effective mass, which yields a critical phase-space density exceeding our experimental conditions.

To conclude, we have experimentally studied the quasicondensate crossover for bilayer excitons confined in a lattice potential. We have observed that a quasicondensate is formed when the barrier height is vanishing, but rapidly destroyed when it is increased to around $0.2 \mathrm{meV}$. We have shown that this behavior is consistent with a renormalization of the exciton effective mass by the lattice depth, which increases the critical phase-space density for the quasicondensation crossover. On the other hand, in the deep lattice limit, we have observed that an array of phase incoherent quasicondensates localized at the lattice sites develops. However, in order to reach the Mott insulator regime, we estimate that the period of the lattice has to be decreased to less than around $1 \mu \mathrm{m}$, such that the effective trapping frequency is increased in the lattice sites. Thus, the strength of on-site interactions is enhanced [1] and becomes a few times larger than the thermal activation energy.

Our work has been financially supported by the Labex Matisse, the Fondation NanoSciences (Grenoble), and by
OBELIX from the French Agency for Research (ANR-15CE30-0020). The work at Princeton University was funded by the Gordon and Betty Moore Foundation through the EPiQS initiative Grant No. GBMF4420, and by the National Science Foundation MRSEC Grant No. DMR 1420541.

[1] M. Greiner, Ph. D. thesis, University of Munich, 2002, https://edoc.ub.uni-muenchen.de/968/.

[2] Many-Body Physics with Ultracold Gases, edited by C. Salomon, G. V. Shlyapnikov, and L. F. Cugliandolo, Lecture Notes of the Les Houches Summer School, Session XCIV Vol. 94 (Oxford University Press, Oxford, 2010).

[3] M. Lewenstein, A. Sanpera, and V. Ahufinger, Ultracold Atoms in Optical Lattices: Simulating Quantum Many-Body Systems (Oxford University Press, Oxford, 2012).

[4] M. Combescot, R. Combescot, and F. Dubin, Rep. Prog. Phys. 80, 066501 (2017).

[5] P. Rivera, H. Yu, K. L. Seyler, N. P. Wilson, W. Yao, and X. Xu, Nat. Nanotechnol. 13, 1004 (2018).

[6] C. Lagoin, S. Suffit, M. Bernard, M. Vabre, K. West, K. Baldwin, L. Pfeiffer, and F. Dubin, Phys. Rev. B 102, 245428 (2020).

[7] M. Remeika, M. M. Fogler, L. V. Butov, M. Hanson, and A. C. Gossard, Appl. Phys. Lett. 100, 061103 (2012).

[8] K. L. Seyler, P. Rivera, H. Yu, N. P. Wilson, E. L. Ray, D. G. Mandrus, J. Yan, W. Yao, and X. Xu, Nature (London) 567, 66 (2019).

[9] K. Tran et al., Nature (London) 567, 71 (2019).

[10] C. Jin et al., Nature (London) 567, 76 (2019).

[11] S. Dang, M. Zamorano, S. Suffit, K. West, K. Baldwin, L. Pfeiffer, M. Holzmann, and F. Dubin, Phys. Rev. Research 2, 032013(R) (2020).

[12] A. A. High, A. K. Thomas, G. Grosso, M. Remeika, A. T. Hammack, A. D. Meyertholen, M. M. Fogler, L. V. Butov, M. Hanson, and A. C. Gossard, Phys. Rev. Lett. 103, 087403 (2009).

[13] G. Grosso, Nat. Photonics 3, 577 (2009).

[14] A. G. Winbow et al., Phys. Rev. Lett. 106, 196806 (2011).

[15] Y. Y. Kuznetsova, P. Andreakou, M. W. Hasling, J. R. Leonard, E. V. Calman, L. V. Butov, M. Hanson, and A. C. Gossard, Opt. Lett. 40, 589 (2015).

[16] I. Rosenberg, Y. Mazuz-Harpaz, R. Rapaport, K. West, and L. Pfeiffer, Phys. Rev. B 93, 195151 (2016).

[17] L. V. Butov, Superlattices Microstruct. 108, 2 (2017).

[18] R. Anankine, M. Beian, S. Dang, M. Alloing, E. Cambril, K. Merghem, C. Gomez Carbonell, A. Lemaître, and F. Dubin, Phys. Rev. Lett. 118, 127402 (2017).

[19] S. Dang, R. Anankine, C. Gomez Carbonell, A. Lemaître, M. Holzmann, and F. Dubin, Phys. Rev. Lett. 122, 117402 (2019).

[20] N. V. Prokofeev and B. V. Svistunov, J. Exp. Theor. Phys. 127, 860 (2018).

[21] M. Combescot and S. Y. Shiau, Excitons and Cooper Pairs: Two Composite Bosons in Many-Body Physics (Oxford University Press, Oxford, 2016).

[22] V. L. Berezinskiǔ, Sov. Phys. JETP 34.3, 610 (1972). 
[23] J. M. Kosterlitz and D. J. Thouless, J. Phys. C 6, 1181 (1973); J. M. Kosterlitz, J. Phys. C 7, 1046 (1974).

[24] M. Naraschewski and R. J. Glauber, Phys. Rev. A 59, 4595 (1999).

[25] M. Beian, M. Alloing, R. Anankine, E. Cambril, C. Gomez Carbonell, A. Lemaître, and F. Dubin, Europhys. Lett. 119, 37004 (2017).

[26] A. Filinov, N. V. Prokof'ev, and M. Bonitz, Phys. Rev. Lett. 105, 070401 (2010).

[27] R. Anankine, S. Dang, M. Beian, E. Cambril, C. Gomez Carbonell, A. Lemaître, and F. Dubin, New J. Phys. 20, 073049 (2018).

[28] A. L. Ivanov, E. A. Muljarov, L. Mouchliadis, and R. Zimmermann, Phys. Rev. Lett. 104, 179701 (2010).

[29] C. Schindler and R. Zimmermann, Phys. Rev. B 78, 045313 (2008).
[30] B. Laikhtman and R. Rapaport, Phys. Rev. B 80, 195313 (2009).

[31] S. V. Andreev, A. A. Varlamov, and A. V. Kavokin, Phys. Rev. Lett. 112, 036401 (2014).

[32] R. M. Bradley and S. Doniach, Phys. Rev. B 30, 1138 (1984).

[33] L. Pitaevskii and S. Stringari, Phys. Rev. Lett. 87, 180402 (2001).

[34] S. Burger, F. S. Cataliotti, C. Fort, P. Maddaloni, F. Minardi, and M. Inguscio, Eur. Phys. Lett. 57, 1 (2002).

[35] S. V. Andreev, Phys. Rev. B 95, 184519 (2017).

[36] F. Dalfovo, L. Pitaevskii, and S. Stringari, Phys. Rev. A 54, 4213 (1996).

[37] M. Holzmann, W. Krauth, and M. Naraschewski, Phys. Rev. A 59, 2956 (1999).

[38] K. Berg-Sorensen and K. Molmer, Phys. Rev. A 58, 1480 (1998). 